\title{
Real-time phase-contrast MRI of cardiovascular blood flow using undersampled radial fast low-angle shot and nonlinear inverse reconstruction
}

\author{
Arun A. Joseph ${ }^{a}$, Klaus-Dietmar Merboldt ${ }^{a}$, Dirk Voit ${ }^{a}$, Shuo Zhang ${ }^{a}$, \\ Martin Uecker ${ }^{a}$, Joachim Lotz ${ }^{b}$ and Jens Frahm ${ }^{a *}$
}

\begin{abstract}
Velocity-encoded phase-contrast MRI of cardiovascular blood flow commonly relies on electrocardiogram-synchronized cine acquisitions of multiple heartbeats to quantitatively determine the flow of an averaged cardiac cycle. Here, we present a new method for real-time phase-contrast MRI that combines flow-encoding gradients with highly undersampled radial fast low-angle shot acquisitions and phase-sensitive image reconstructions by regularized nonlinear inversion. Apart from calibration studies using steady and pulsatile flow, preliminary in vivo applications focused on through-plane flow in the ascending aorta of healthy subjects. With bipolar velocity-encoding gradients of alternating polarity that overlap the slice-refocusing gradient, the method yields flow-encoded images with an in-plane resolution of $1.8 \mathrm{~mm}$, section thickness of $6 \mathrm{~mm}$ and measuring time at $3 \mathrm{~T}$ of $24 \mathrm{~ms}$ (TR/TE = 3.44/2.76 ms; flip angle, $10^{\circ}$; seven radial spokes per image). Accordingly, phase-contrast maps and corresponding velocity profiles achieve a temporal resolution of $\mathbf{4 8} \mathrm{ms}$. The evaluated peak velocities, stroke volumes, flow rates and respective variances over at least 20 consecutive heartbeats are in general agreement with literature data. Copyright @ 2011 John Wiley \& Sons, Ltd. Supporting information may be found in the online version of this paper
\end{abstract}

Keywords: cardiovascular MRI; flow imaging; heart function; nonlinear inverse reconstruction; phase-contrast imaging; real-time imaging

\section{INTRODUCTION}

Velocity-encoded phase-contrast MRI is a well-established method for quantitative mapping of flow velocities. It is based on Erwin Hahn's 1960 observation of a phase shift for spins moving in the presence of a magnetic field gradient (1), and has been developed during the early stages of MRI [see, for example, refs. (2-5)]. In its simplest form, quantitative velocities of a constant flow may be derived from the phase difference between two cross-sectional images with different velocity-encoding gradients (e.g. two bipolar gradients with opposite polarity). The resulting three-dimensional dataset comprises two spatial dimensions and one velocity (phase-difference) dimension, so that each image pixel presents with a single mean velocity value. Applications to clinical studies of the human heart and great vessels have been reviewed several times [see, for example, refs. (6-8)].

In order to obtain sufficient temporal resolution during the cardiac cycle, MRI studies commonly rely on electrocardiogram (ECG)-synchronized cine acquisitions of multiple heartbeats, either during free breathing or in suspended respiration. The approach may be affected by an inappropriate temporal and/or spatial averaging of phase information as a result of the calculation of a phase-difference value from the complex mean of signals of multiple spins (9), and therefore bears the risk of underestimating maximum flow velocities compared with, for example, Doppler sonography. Moreover, it renders the technique susceptible to flow inconsistencies caused by cardiac arrhythmias. Indeed, the direct assessment of physiological variability or pathological irregularities, such as arrhythmia, is not feasible with acquisitions extending over several heartbeats.

Many ideas have been proposed to ameliorate the detrimental effects of motion in MRI. In order to allow for free breathing in cardiovascular applications, the most successful attempts range from navigator-type corrections based on concentric rectangular strips or blades rotated about the center of $k$ space (10) to the use of respiratory self-gating (11). A similar wide range of methods has been proposed for real-time MRI since the early work by Chapman et al. (12) using echo-planar imaging. The approaches employ low-flip-angle radiofrequency (RF) excitations of gradient echoes and cover $k$ space with the use of an echo-planar (13), Cartesian (14-16), radial $(17)$ or spiral $(18,19)$ trajectory. In recent

* Correspondence to: J. Frahm, Biomedizinische NMR Forschungs GmbH am Max-Planck-Institut für biophysikalische Chemie, 37070 Göttingen, Germany. E-mail: jfrahm@gwdg.de

a A. A. Joseph, K.-D. Merboldt, D. Voit, S. Zhang, M. Uecker, J. Frahm Biomedizinische NMR Forschungs $\mathrm{GmbH}$ am Max-Planck-Institut für biophysikalische Chemie, Göttingen, Germany

b J. Lotz

Abteilung Diagnostische Radiologie, Universitätsklinikum, Göttingen, Germany

Abbreviations used: ECG, electrocardiogram; FLASH, fast low-angle shot; FOV, field of view; GRAPPA, generalized autocalibrating partially parallel acquisition; RF, radiofrequency; SD, standard deviation; SENSE, sensitivity encoding. 
years, further acceleration has been achieved by combinations with parallel imaging based on sensitivity encoding (SENSE) (2023) or generalized autocalibrating partially parallel acquisition (GRAPPA) (24), or by further exploiting temporal continuities (25). Nevertheless, the hitherto still limited spatiotemporal resolution and/or overall image quality of many proposed techniques must be considered responsible for a lack of general clinical acceptance.

Most recently, we have made significant advances in highquality real-time MRI using highly undersampled radial fast low-angle shot (FLASH) acquisitions with image reconstruction by regularized nonlinear inversion (26-28). Preliminary applications to cardiovascular MRI allowed for serial real-time images (i.e. movies) with an in-plane resolution of $1.5-2.0 \mathrm{~mm}$ and an acquisition time of $20-30 \mathrm{~ms}(28,29)$. Thus, the purpose of this study was to combine the principles of velocity-encoded phase-contrast MRI with the acquisition of two highly undersampled radial FLASH images to achieve a temporal resolution for real-time flow MRI of less than $50 \mathrm{~ms}$.

\section{METHODS}

\section{SUBJECTS}

Apart from pilot studies that were performed to optimize the experimental conditions and define the measurement protocol, a total of six healthy male subjects without prior cardiovascular symptoms and no known illness participated in the real-time assessment of through-plane flow in the ascending aorta. The deliberately chosen heterogeneous group of subjects presented with different ages (range, 16-57 years), body weights and physical constitutions. All subjects gave written informed consent before each MRI examination.

\section{Velocity-encoded phase-contrast MRI in real time}

All studies were conducted at $3 \mathrm{~T}$ using a commercial MRI system (TIM Trio, Siemens Healthcare, Erlangen, Germany) and a body coil for RF excitation. Subjects were examined in a supine position and $\mathrm{MRI}$ signals were acquired with the use of a 32-channel cardiac coil comprising an anterior and posterior 16-element array.

Real-time phase-contrast MRI employed a highly undersampled RF-spoiled radial FLASH sequence (30) with a motioncompensated slice-selective gradient and pairs of flow-encoding gradients in two interleaved acquisitions (every other TR). Velocity encoding to measure through-plane flow was implemented along the slice-selective gradient axis in two different ways. As indicated in Fig. 1, the first scheme employed bipolar velocity-encoding gradients with opposite polarity (Min-Max version), and the second scheme applied no flow-encoding gradient during one acquisition and a correspondingly stronger velocity-encoding gradient during the other (Zero-Max version, not shown). In either case, the field of view (FOV) in the phase-contrast or velocity dimension was set to $\mathrm{VENC}=150 \mathrm{~cm} / \mathrm{s}$. The velocity-encoding gradients were combined with the refocusing part of the slice-selective gradient, as described previously for conventional cine techniques (31). However, the actual implementation did not realize a further overlap with the two frequency-encoding gradients to retain more flexibility for the incorporation of flow-encoding gradients along arbitrary directions.

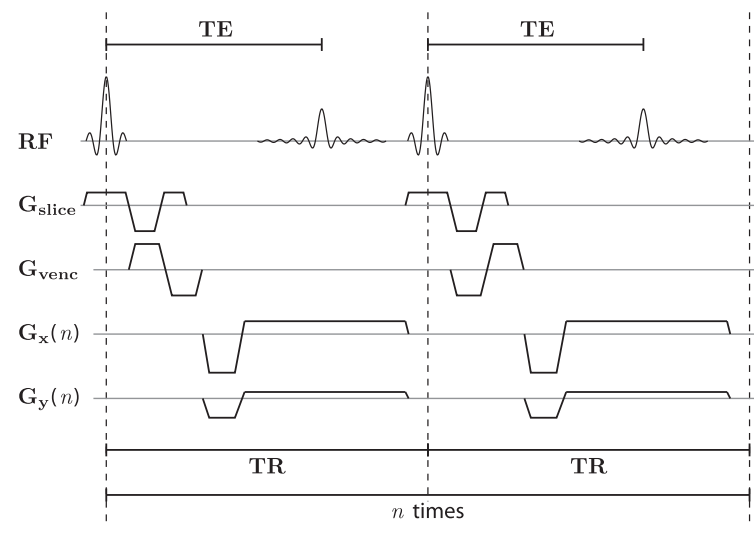

Figure 1. Schematic radiofrequency (RF) pulse and gradient sequence for real-time velocity-encoded phase-contrast MRI of through-plane flow. The method is based on highly undersampled radial fast low-angle shot $(\mathrm{FLASH})\left(\mathrm{TR} / \mathrm{TE}=3.44 / 2.76 \mathrm{~ms}\right.$; flip angle, $\left.10^{\circ}\right)$ and relies on two interleaved image acquisitions ( $n=7$ spokes per image) with bipolar velocity-encoding gradients $G_{\text {VENC }}$ of opposite polarity (VENC $=150$ $\mathrm{cm} / \mathrm{s}$ ). These gradients are combined with the refocusing part of the motion-compensated slice-selective gradient $G_{\text {slice, }}$ but do not overlap with the frequency-encoding gradients $G_{x}$ and $G_{y}$ to allow for additional flow-encoding gradients along arbitrary directions. The implementation yields a temporal resolution of $48 \mathrm{~ms}$ per phase-contrast map.

Gradient switching times were kept to a normal (rather than fast) mode, which refers to slew rates below the technical possibilities of the MRI system used. This choice avoids peripheral nerve stimulation under all experimental circumstances. Accordingly, the Zero-Max version resulted in minimum values of $\mathrm{TR} / \mathrm{TE}=3.15 / 2.46 \mathrm{~ms}$ and a temporal resolution of $44 \mathrm{~ms}$ for phase-contrast MRI. The corresponding results for the Min-Max version were $\mathrm{TR} / \mathrm{TE}=3.44 / 2.76 \mathrm{~ms}$ and a resolution of $48 \mathrm{~ms}$.

All real-time applications relied on image acquisitions with only seven spokes (144 complex data points each) covering a FOV of $256 \times 256 \mathrm{~mm}^{2}$ at an in-plane resolution of $1.8 \mathrm{~mm}$ and with a slice thickness of $6 \mathrm{~mm}$ (flip angle, $10^{\circ}$ ). Successive acquisitions employed five different sets of complementary spokes, as described previously (30). Their purpose is twofold: first, the strategy generates slightly different streaking artifacts from frame to frame that are more easily treatable than constant artifacts and, second, combined datasets (i.e. 35 spokes) are exploited for immediate online control during scanning using gridding reconstructions (see below). In our current setting, these images are replaced within a few minutes by the respective inverse reconstructions performed offline.

\section{Image reconstructions}

Fully sampled radial FLASH images served as multislice localizer scans, as well as for comparative phantom studies. Such images were reconstructed by conventional gridding. Similarly, online monitoring of velocity-encoded magnitude images and phase-contrast maps during real-time flow MRI was accomplished by gridding of combined datasets with 35 spokes and a sliding window factor of five. Thus, these gridding images cover a five-fold longer acquisition time than the respective nonlinear inverse reconstructions, but offer the same frame rate. Although partially compromised by streaking artifacts and enhanced noise, such reconstructions provide sufficient quality to supervise the actual measurement. In order to gain real-time speed, gridding 
reconstructions employed an online channel compression (30) based on a principal component analysis of the first few scans. It reduces the data from the 32-channel acquisition to the most relevant six or eight components ('virtual channels') and thus ensures sufficient reconstruction speed for immediate visual control.

For offline reconstruction using regularized nonlinear inversion, the incoming data were automatically exported to a computer equipped with eight GTX580 graphical processing units, each providing 512 processing cores (Nvidia, Santa Clara, CA, USA). Extending the original algorithm to jointly estimate image content and coil sensitivity profiles (19) and its adaptation to real-time imaging $(27,28)$, the present framework allows for the reconstruction of phase-sensitive images before taking a phase-difference map. Although still applying a temporal median filter to the reconstructed magnitude images (i.e. to reduce residual streaking artifacts), the phase-contrast maps are calculated from the unfiltered complex-valued images. To remove unwanted phase components from coil sensitivities or off-resonance effects, each reconstructed image was multiplied with all coil sensitivities to obtain separate images for all channels. Phase-difference maps were then calculated for each individual channel by multiplying the first flow-encoded image with the complex conjugate of the associated second flow-encoded image. The final phase-contrast map was determined by summing the results of all channels and extracting the phase (32), which also ensures that contributions from individual channels are weighted with their respective energy in each pixel.

Once the offline calculation of a real-time MRI series had been completed, the magnitude images and corresponding phasecontrast maps were re-imported into the MRI scanner by exploiting the manufacturer's mechanism of a 'retrospective reconstruction'. However, instead of again reconstructing the raw data which are temporally stored on the scanner, the formalism was used to create DICOM images with the correct header information from the offline reconstructions for storage in the system's databank. To date, where offline reconstructions still take some time, this strategy provides a simple and almost fully automatic by-pass of the normal reconstruction process with only a single manual intervention (i.e. starting the re-import on the scanner). In fact, most nonlinear inverse reconstructions of a real-time heart study are already available after the end of the examination, so that the images may immediately be re-imported onto the scanner for further evaluation.

The total reconstruction time for two flow-encoded images and the corresponding phase-contrast map is currently about $0.2 \mathrm{~s}$ using four parallel graphical processing units and a grid size

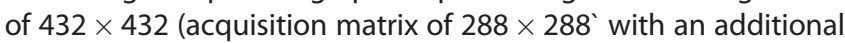
oversampling factor of 1.5 for reconstruction). This includes a reduction of the original 32-channel dataset to 12 virtual channels by a principal component analysis and the use of six Newton steps for the iterative optimization (28).

\section{Flow calibration}

Although commercial phase-contrast flow MRI sequences employ similar, if not identical, combinations of flow-encoding and imaging gradients, we performed independent calibration experiments to evaluate the accuracy of the entire acquisition and analysis process, and to ascertain the equivalence of phase-contrast maps that were obtained by gridding of fully sampled datasets with those calculated by nonlinear inversion of highly undersampled datasets.
Flow calibrations used a 16-mm-diameter stiff tube to simulate the properties of the aorta. Flow at different rates was provided by a computer-controlled immersion pump (Lux Plus KTW270, Herzog, Göttingen, Germany) and independently measured by an external flow meter (FCH-C-PA, BioTech, Vilshofen, Germany) outside the main magnetic field of the scanner. Studies of steady flow were performed with both the Zero-Max and Min-Max flowencoding schemes at temporal resolutions of 44 and $48 \mathrm{~ms}$, respectively.

Pulsatile flow was generated by a repetitive pump protocol with brief periods of high velocity $(0.1-0.4 \mathrm{~ms})$, followed by a lower velocity for a total cycle time of $2.0 \mathrm{~s}$. High- and low-flow conditions referred to pump voltages differing by a factor of two. Respective phase-contrast MRI studies were performed with the Min-Max encoding scheme.

\section{Human studies}

Because the calibration measurements resulted in a lower phase offset for stationary spins when using the Min-Max version (see Results), all real-time phase-contrast MRI studies of the ascending aorta were performed with polarity-alternated bipolar velocity-encoding gradients at a temporal resolution of $48 \mathrm{~ms}$ in a transverse orientation. These real-time acquisitions were accompanied by ECG recordings to provide an ECG stamp, i.e. the relative time to the last $R$ wave, to each magnitude image and phase-contrast map.

The total measurement protocol comprised real-time phasecontrast MRI movies during free breathing (about $30 \mathrm{~s}$ ), as well as during breath hold (about 15s), in inspiration and expiration. Three free-breathing studies were interleaved with two repetitions of two breath hold scans (reversed order of inspiration and expiration), yielding a total of seven experimental runs. Pauses between scans were at least $30 \mathrm{~s}$. The total examination time, including scans for positioning (sequential multislice radial FLASH MRI), was about $15 \mathrm{~min}$.

\section{Flow analysis}

All flow-encoded phase-contrast maps were analyzed using ARGUS supplied by the manufacturer (Siemens Healthcare, Erlangen, Germany). In addition, human flow MRI data were also evaluated by QFlow 5.2 (Medis, Leiden, the Netherlands) which offers greater flexibility and extended parametric access. Both programs define a region of interest by complementing a manual outline of the aorta in a single magnitude image with an automatic segmentation and propagation to all images and maps of a series. In human studies, the automatic segmentation by QFlow 5.2 was visually controlled and manually corrected if necessary.

Of note, instead of looking at a single synthetic cardiac cycle from an ECG-synchronized cine recording (i.e. about 20 images), the present analyses comprised 15-20 consecutive heartbeats (each with about 20 images). Although this procedure offers access to the physiological variance of flow parameters from heartbeat to heartbeat, it is also possible to combine all (about 300-400) images into a single dataset with individual images sorted according to the ECG. At least for ARGUS, however, this combination strategy turned out to be more vulnerable to segmentation errors.

The main flow parameters derived from real-time phasecontrast MRI movies were the maximum 'peak' velocity (for ARGUS: mean of one pixel and four neighbors), the stroke volume defined as the output of the left ventricle into the ascending aorta during 
one cardiac cycle, and the flow rate as the mean cardiac output per minute. In contrast with conventional cine recordings, where, for example, the latter parameter must be extrapolated from a single synthetic cardiac cycle to a full minute, real-time evaluations of much longer series of heartbeats allow for more accurate estimates. In fact, it is even possible to extend the measurement to a minute. Finally, color-coded visualization of velocity distributions within the ascending aorta was accomplished using routines written in MATLAB (MathWorks, Natick, MA, USA).

\section{RESULTS AND DISCUSSION}

\section{Flow calibration}

During experimental optimization of the flow-encoding radial FLASH MRI sequences, the residual phase error for stationary spins, i.e. a nonzero mean signal in a stationary region of a phasecontrast map, turned out to be three times smaller for the Min-Max than for the Zero-Max version. A likely explanation for this smaller phase offset is the larger similarity of the overlapping slice-refocusing and flow-encoding gradients when using two polarity-alternated bipolar gradients of equal strength. However, the Zero-Max scheme may lead to a larger disparity, so that residual gradient imperfections (e.g. as a result of eddy currents) are less well compensated in a phase-difference map. When averaging over an area comparable with that of the human aorta, the observed phase offsets corresponded to about $0.38 \mathrm{~L} / \mathrm{min}$ for the Zero-Max and only $0.12 \mathrm{~L} / \mathrm{min}$ for the Min-Max version. Thus, at the expense of a slightly longer acquisition time, we therefore decided to exclusively use bipolar velocity-encoding gradients with opposite polarity for human applications. Moreover, the results presented here are not corrected for residual phase offset.

Figure 2 summarizes the results of a Min-Max calibration study for a 16-mm-diameter flow phantom with constant flow at different rates. The recordings of a flow meter are compared with MRI flow rates derived from gridding reconstructions of fully sampled radial FLASH images, as well as nonlinear inverse reconstructions of highly undersampled acquisitions, with identical parameters as in the human studies, at a resolution of $48 \mathrm{~ms}$. In order to apply the same evaluation procedure as in vivo, the images were labeled with a simulated ECG signal and analyzed by ARGUS. The results demonstrate excellent agreement of MRI and flow meter values for the range of $4-8 \mathrm{~L} / \mathrm{min}$. The observation of a slight overestimation for the lower values of $2-3 \mathrm{~L} / \mathrm{min}$ may be explained by the fact that voxels in the border region experience a disproportionate signal increase from moving spins compared with the contribution from stationary spins (33). Of note, there seems to be no difference between the two types of acquisition, so that real-time MRI studies based on nonlinear inverse reconstructions of highly undersampled datasets may be performed with the same accuracy as conventional phase-contrast recordings.

The calibration studies were complemented by real-time phase-contrast MRI of pulsatile flow generated by protocols with two different velocities (as controlled by the pump voltage) and different durations for a total cycle time of $2.0 \mathrm{~s}$ (same phantom as above). The protocols and resulting flow parameters are summarized in Table 1. Although no independent assessment of the dynamic flow velocities was available, the estimated flow rates from four consecutive cycles are again in excellent agreement with the experimentally determined values. The calibration results for both steady and pulsatile flow therefore confirm the quantitative reliability of the proposed real-time phase-contrast MRI technique for the measurement of flow rates.

\section{Human studies}

Focusing on through-plane flow in the ascending aorta, this preliminary real-time flow MRI study was successful in all subjects. The data were not affected by putative motion problems caused by free breathing, although it cannot be excluded that future applications to other vessels may require more sophisticated strategies that detect respiratory variations directly from the data. All real-time phase-contrast MRI data (six subjects, seven runs each, 15-20 heartbeats each, about 5000 images and phase-contrast maps) were analyzed with both ARGUS and QFlow 5.2 (not shown) and revealed excellent agreement.

Figure 3 shows a selected magnitude image and corresponding phase-contrast map of the ascending aorta from a real-time movie acquisition (temporal resolution of $48 \mathrm{~ms}$ ) at a time point with

\section{Gridding}

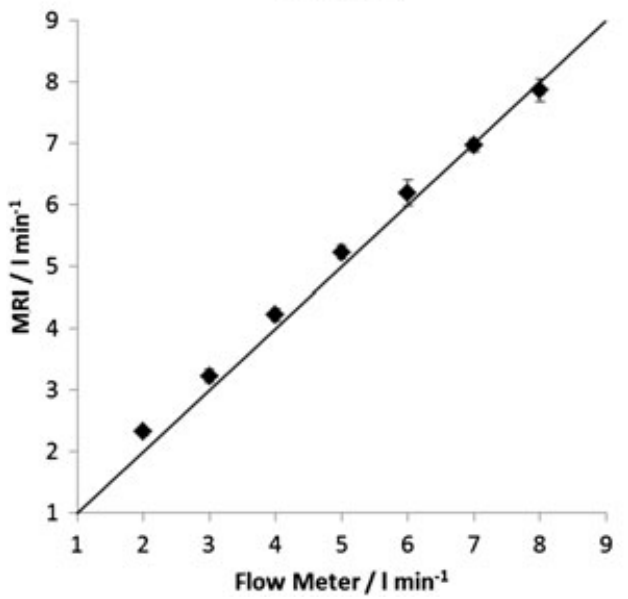

\section{Nonlinear Inversion}

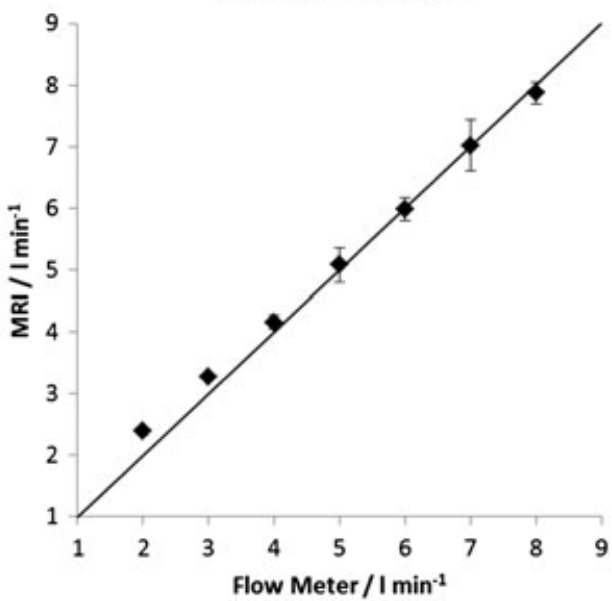

Figure 2. Flow rates (L/min) for a calibration phantom (16-mm-diameter tube) with constant flow measured by phase-contrast MRI (VENC $=150 \mathrm{~cm} / \mathrm{s}$ ) using either fully sampled radial fast low-angle shot (FLASH) with gridding (225 spokes, other parameters as in Fig. 1) or undersampled acquisitions (seven spokes) with nonlinear inverse reconstruction. Calibration values refer to an external flow meter (accuracy, $\pm 0.05 \mathrm{~L} / \mathrm{min}$ ). MRI flow rates represent the mean \pm standard deviation (SD) of data from 10 simulated heartbeats. The diagonal line refers to exact calibration. 


\begin{tabular}{|c|c|c|c|c|c|}
\hline \multicolumn{2}{|c|}{ Repetitive protocol } & \multicolumn{2}{|c|}{ Flow velocity $(\mathrm{cm} / \mathrm{s})$} & \multicolumn{2}{|c|}{ Flow rate (L/min) } \\
\hline High flow & Low flow & High flow & Low flow & MRI & Exp. \\
\hline $0.4 \mathrm{~s}$ & $1.6 \mathrm{~s}$ & $72.2 \pm 0.3$ & $49.7 \pm 0.5$ & $5.35 \pm 0.03$ & 5.30 \\
\hline $0.2 \mathrm{~s}$ & $1.8 \mathrm{~s}$ & $58.2 \pm 2.0$ & $44.4 \pm 0.2$ & $4.63 \pm 0.08$ & 4.63 \\
\hline $0.1 \mathrm{~s}$ & $1.9 \mathrm{~s}$ & $52.5 \pm 1.1$ & $44.4 \pm 0.1$ & $4.38 \pm 0.07$ & 4.47 \\
\hline
\end{tabular}
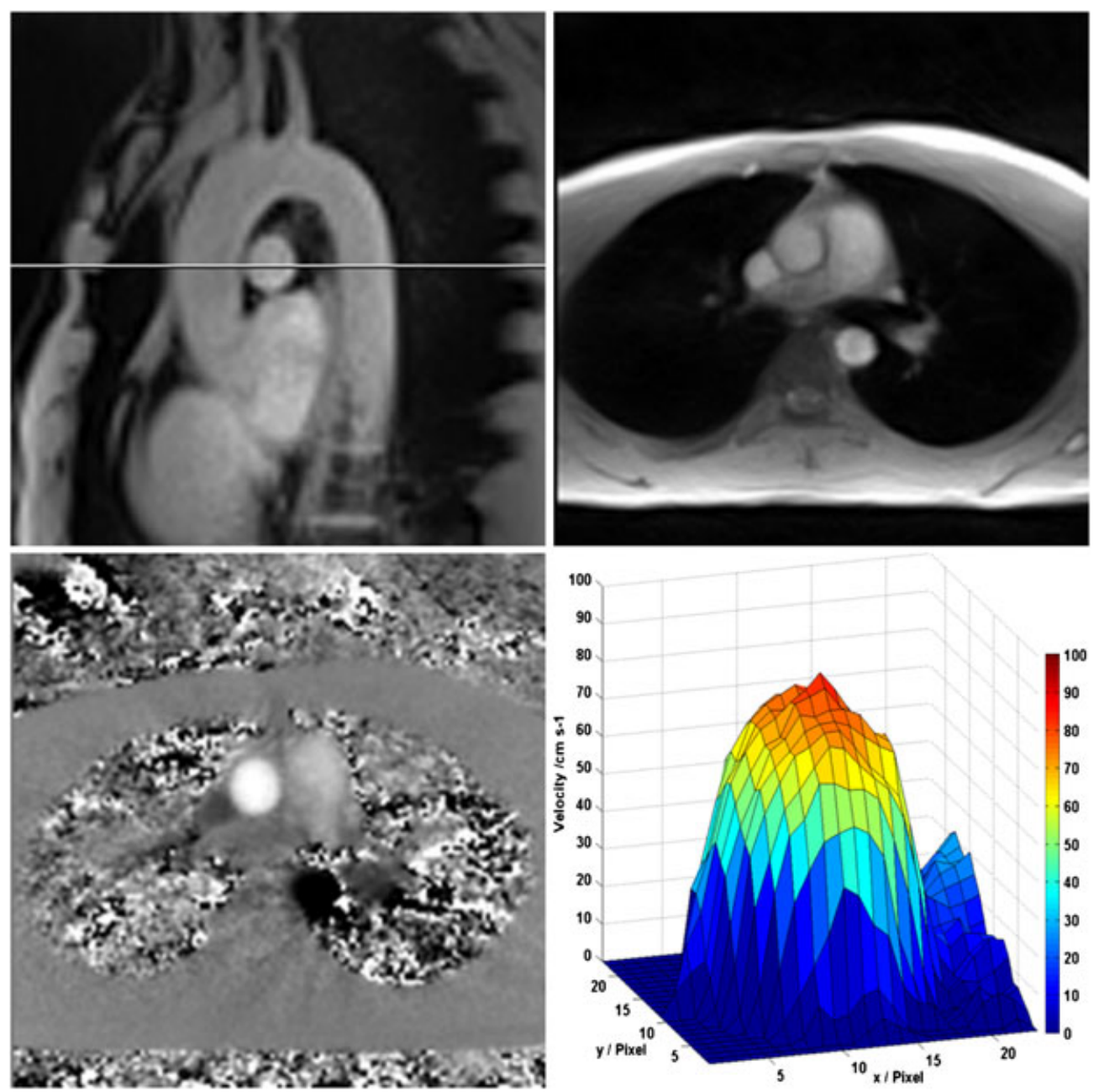

Figure 3. Real-time velocity-encoded MRI of through-plane flow in the ascending aorta (resolution, $48 \mathrm{ms)}$ of a healthy subject. Top left: aortic arch with transverse slice orientation [radial fast low-angle shot (FLASH) localizer]. Top right: selected magnitude image, as well as corresponding phase-contrast map (bottom left) and flow velocities within the aorta (bottom right) (color-coded range from 0 to $100 \mathrm{~cm} / \mathrm{s}$ ). Other parameters as in Fig. 1.

strong systolic flow. In general, the image quality is surprisingly good in view of the extreme undersampling by a factor of 32 , that is seven spokes relative to a formally required number of $144 \times \pi /$ $2=226$ spokes. In particular, the phase-contrast map is only marginally affected by residual streaking artifacts (outside the area of interest), and the phase differences for stationary spins are rather homogeneous and close to zero. A dynamic representation of phase-contrast maps from 10 consecutive heartbeats of this dataset is available as Supporting Information (Movie S1). The bottom right map in Fig. 3 visualizes the two-dimensional flow profile within the ascending aorta by color-coded velocities ranging from 0 (blue) to $100 \mathrm{~cm} / \mathrm{s}$ (red).

Figure 4 depicts the dynamic velocity distributions of flow in the ascending aorta at a resolution of $48 \mathrm{~ms}$. The series of 12 consecutive profiles (total duration, $576 \mathrm{~ms}$ ) covers the early phase of a single cardiac cycle. In this subject, the velocity increases to a peak value of $80 \mathrm{~cm} / \mathrm{s}$ (red) within about $150 \mathrm{~ms}$ (three frames). Its slightly slower decline is accompanied by the 

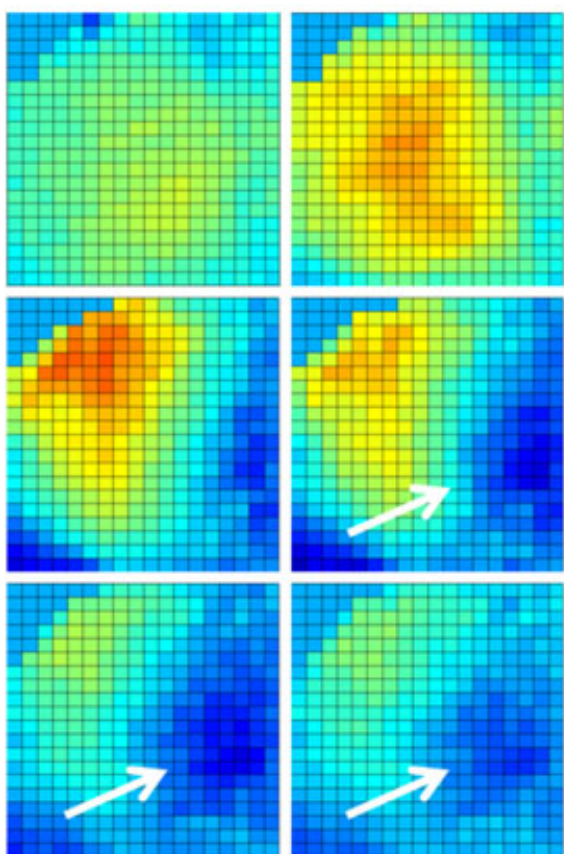
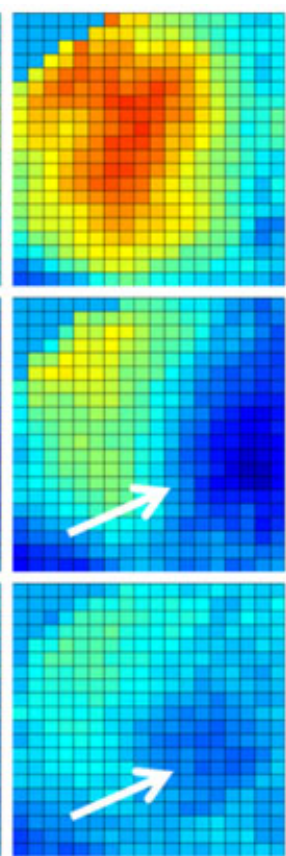
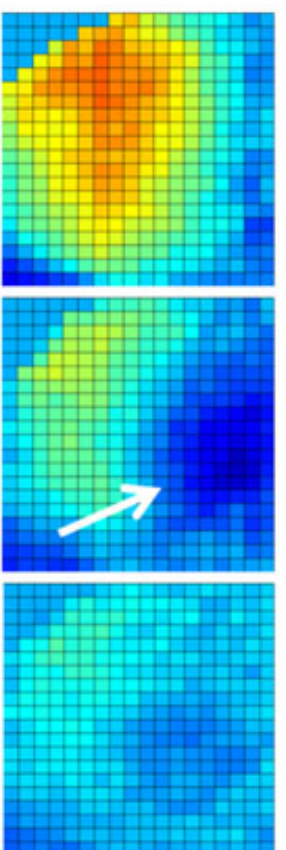

100

80

60

$40 \stackrel{\text { in }}{\frac{\text { in }}{0}}$

0

$-20$

$-40$

Figure 4. Real-time flow velocities (color-coded range from -40 to $100 \mathrm{~cm} / \mathrm{s}$ ) within the ascending aorta ( $18 \times 20$ pixels) at 12 consecutive time points during the early phase of a single cardiac cycle (resolution, $48 \mathrm{~ms}$; total duration, $12 \times 48 \mathrm{~ms}=576 \mathrm{~ms}$; different subject from Fig. 3 ). Arrows indicate back flow (blue). Other parameters as in Fig. 1.

occurrence of back flow with velocities of up to $40 \mathrm{~cm} / \mathrm{s}$ (arrows, dark blue). A dynamic visualization of real-time velocity profiles from 20 consecutive heartbeats is available as Supporting information (Movie S2).

Figure 5 demonstrates the variability of peak flow velocities and stroke volumes as a function of time (20 cardiac cycles) under the condition of free breathing. The lower portions of Fig. 5 contain temporal signal intensity profiles of the anterior chest wall from corresponding magnitude images that indicate inspiration (arrows) and expiration phases. Although peak velocities vary during the respiratory cycle (here about four to five cardiac cycles) by more than $10 \%$ between expiration and inspiration, the stroke volume remains more stable. The results indicate that the lowest peak velocities are obtained during expiration.
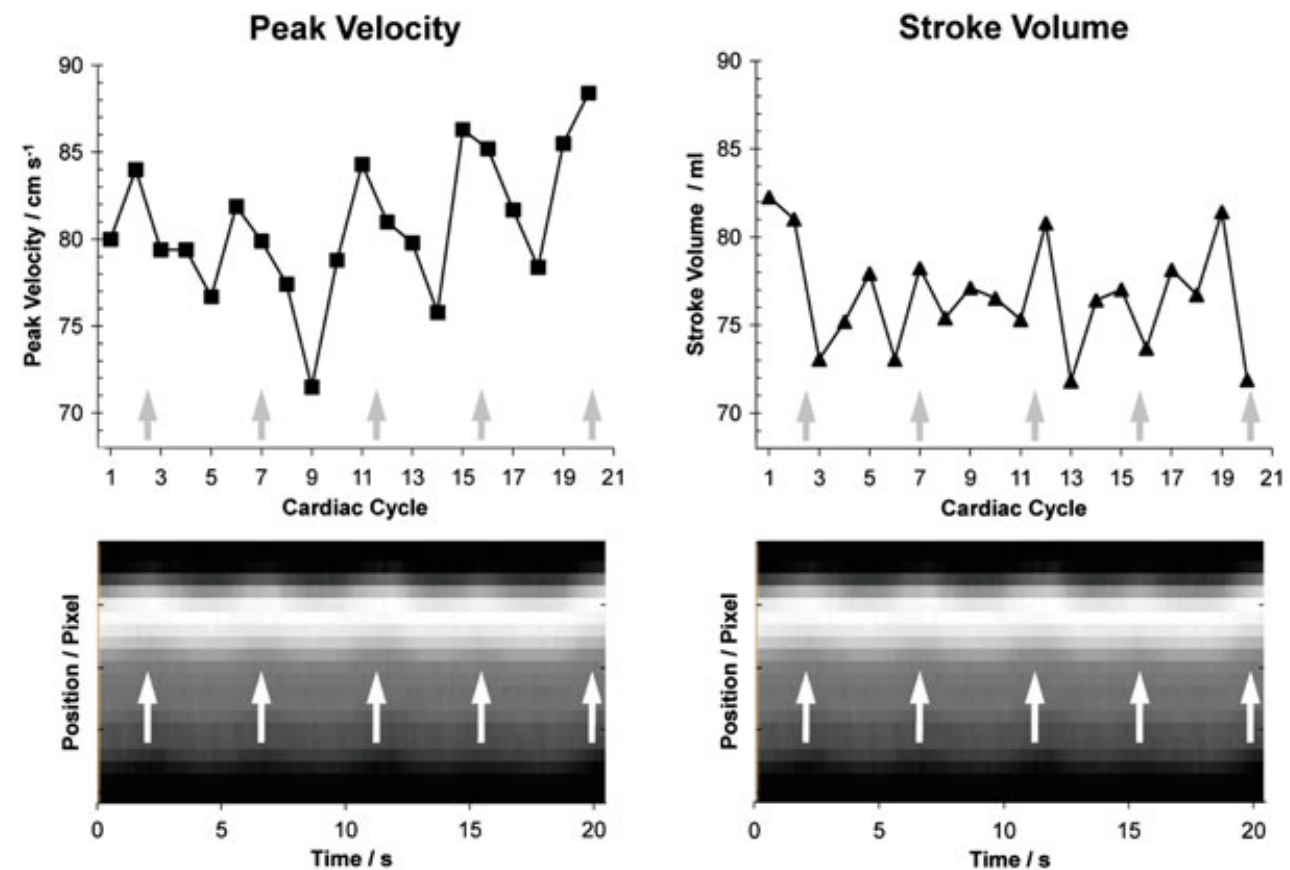

Figure 5. Real-time peak flow velocities $(\mathrm{cm} / \mathrm{s})$ in the ascending aorta and related stroke volumes $(\mathrm{mL})$ over 20 consecutive heartbeats (resolution, $48 \mathrm{~ms}$; same subject as in Fig. 3). The bottom parts refer to temporal signal intensity profiles of the anterior chest wall taken from the corresponding magnitude images. Arrows indicate inspiration. Other parameters as in Fig. 1. 
Quantitative results for all subjects are summarized in Table 2 for free breathing. All flow parameters [mean \pm standard deviation (SD)] are averaged across 20 heartbeats and three repetitions (corresponding to 60 heartbeats). The observed differences for the peak velocity between subjects most probably represent the physiological variation of hemodynamics in response to parameters such as, for example, body composition, blood pressure and state or alertness during MRI. As such, our small group of subjects varied considerably with respect to age, body weight and physical fitness, so that individual variances (i.e. intrasubject SD values) for peak velocities, stroke volumes and flow rates are much smaller than the differences between the corresponding mean values of individual subjects.

Mean flow parameters averaged across subjects are shown in Table 3 for free breathing and breath holding under inspiration and expiration. Peak velocities in the ascending aorta tend to be higher during breath hold than during free breathing, whereas stroke volumes remain remarkably constant. The former observation matches the finding of a respiratory-dependent variation of peak velocities as seen in Fig. 5. Both absolute and individually normalized flow rates increase during expiration, as expected for a lowered intrathoracic pressure. The considerably larger SD value for the stroke volume under breath hold with inspiration is most probably a result of an inconsistent performance of our subjects.

Altogether, the flow parameters for free breathing seem to better reflect a normal physiological state than those obtained during breath hold. This observation is supported by rather small SD values for peak velocities and flow rates under free breathing, despite the heterogeneity of the subjects. Larger studies are needed to confirm these initial findings.

\section{CONCLUSIONS}

This work describes the development and implementation of a real-time method for velocity-encoded phase-contrast MRI with a temporal resolution below $50 \mathrm{~ms}$. Calibration studies confirmed the accuracy of flow rates obtained from highly undersampled radial FLASH acquisitions with nonlinear inverse reconstruction. The evaluation of real-time flow parameters for the ascending aorta of healthy human subjects revealed general agreement with literature data: for example, compare refs. $(34,35)$ using MRI and non-MRI techniques. Moreover, the present results support the notion that free breathing is a preferred condition for the physiological characterization of cardiovascular flow. It is expected that real-time access to flow parameters (over any chosen period of time) will offer new potential for clinical assessments of pathological deviations or irregularities within single or serial heartbeats.

Although beyond the scope of this methodological work, a limitation of our study is the small number of subjects and the restriction to flow in the ascending aorta. A clinical evaluation of real-time flow measurements in direct comparison with ECG-gated phase-contrast MRI will address this shortcoming in the near future. Another restriction that needs to be taken into account is the available software for flow analysis. Although existing packages may be applicable, as used here, suitable adjustments will considerably accelerate the processing of data from a large number of heartbeats and more easily provide information about physiological or pathological variations. Pertinent improvements will be a prerequisite for the clinical acceptance of real-time flow MRI examinations.

Table 2. Flow parameters for the ascending aorta of six healthy subjects as determined by real-time velocity-encoded phasecontrast MRI during free breathing

\begin{tabular}{|c|c|c|c|c|c|c|}
\hline Subject & Age (years) & $\begin{array}{l}\text { Heart rate } \\
\text { (beats/min) }\end{array}$ & $\begin{array}{l}\text { Peak velocity } \\
(\mathrm{cm} / \mathrm{s})\end{array}$ & $\begin{array}{c}\text { Stroke } \\
\text { volume }(\mathrm{mL})\end{array}$ & $\begin{array}{c}\text { Aortic } \\
\text { area }\left(\mathrm{cm}^{2}\right)\end{array}$ & $\begin{array}{c}\text { Flow rate } \\
\text { (L/min) }\end{array}$ \\
\hline 1 & 24 & $62 \pm 2$ & $81 \pm 9$ & $102 \pm 8$ & $6.9 \pm 0.3$ & $6.4 \pm 0.5$ \\
\hline 2 & 37 & $60 \pm 4$ & $73 \pm 6$ & $97 \pm 13$ & $6.9 \pm 0.5$ & $5.8 \pm 0.8$ \\
\hline 3 & 57 & $68 \pm 3$ & $54 \pm 3$ & $96 \pm 9$ & $10 \pm 0.4$ & $6.5 \pm 0.6$ \\
\hline 4 & 28 & $64 \pm 4$ & $68 \pm 4$ & $74 \pm 9$ & $6.4 \pm 0.2$ & $4.7 \pm 0.6$ \\
\hline 5 & 22 & $89 \pm 7$ & $59 \pm 4$ & $68 \pm 10$ & $6.1 \pm 0.4$ & $6.0 \pm 0.9$ \\
\hline 6 & 25 & $57 \pm 3$ & $79 \pm 6$ & $76 \pm 4$ & $5.2 \pm 0.3$ & $4.3 \pm 0.2$ \\
\hline
\end{tabular}

Values are given as mean \pm standard deviation (SD) averaged across 20 heartbeats and three repetitions (i.e. a total of 60 heartbeats).

Table 3. Mean flow parameters for the ascending aorta as determined by real-time velocity-encoded phase-contrast MRI during free breathing as well as breath hold in inspiration and expiration

\begin{tabular}{|c|c|c|c|c|}
\hline Breathing condition & $\begin{array}{l}\text { Peak velocity } \\
\qquad(\mathrm{cm} / \mathrm{s})\end{array}$ & $\begin{array}{l}\text { Stroke volume } \\
\qquad(\mathrm{mL})\end{array}$ & $\begin{array}{l}\text { Flow rate } \\
\text { (L/min) }\end{array}$ & $\begin{array}{c}\text { Normalized flow } \\
\text { rate (\%) }\end{array}$ \\
\hline Free & $68.8 \pm 10.6$ & $85.7 \pm 13.6$ & $5.6 \pm 0.9$ & 100 \\
\hline Inspiration & $73.9 \pm 15.0$ & $87.4 \pm 23.6$ & $5.8 \pm 1.3$ & $102 \pm 9$ \\
\hline Expiration & $74.5 \pm 15.6$ & $85.3 \pm 11.9$ & $6.1 \pm 1.1$ & $109 \pm 15$ \\
\hline
\end{tabular}




\section{REFERENCES}

1. Hahn EL. Detection of sea-water motion by nuclear precession. J. Geophys. Res. 1960; 65: 776-777.

2. Moran PR. A flow velocity zeugmatographic interlace for NMR imaging in humans. Magn. Reson. Imaging, 1982; 1: 197-203.

3. Van Dijk P. Direct cardiac NMR imaging of heart wall and blood flow velocity. J. Comput. Assist. Tomogr. 1984; 8: 429-436.

4. Bryant DJ, Payne JA, Firmin DN, Longmore DB. Measurement of flow with NMR imaging using a gradient pulse and phase difference technique. J. Comput. Assist. Tomogr. 1984; 8: 588-593.

5. Nayler GL, Firmin DN, Longmore DB. Blood flow imaging by cine magnetic resonance imaging. J. Comput. Assist. Tomogr. 1986; 10: 715-722.

6. Pelc NJ, Herfkens RJ, Shimakawa A, Enzmann DR. Phase contrast cine magnetic resonance imaging. Magn. Reson. Q. 1991; 7: 229-254.

7. Lotz J, Meier C, Leppert A, Galanski M. Cardiovascular flow measurement with phase-contrast MR imaging: basic facts and implementation. Radiographics 2002; 22: 651-671.

8. Gatehouse PD, Keegan J, Crowe LA, Masood S, Mohiaddin RH, Kreitner KF, Firmin DN. Applications of phase-contrast flow and velocity imaging in cardiovascular MRI. Eur. Radiol. 2005; 15: 2172-2184.

9. Bernstein MA, King KF, Zhou XJ. Handbook of MRI Pulse Sequences. Elsevier: Amsterdam; 2004; 666-672.

10. Pipe JG. Motion correction with PROPELLER MRI: application to head motion and free-breathing cardiac imaging. Magn. Reson. Med. 1999; 42: 963-969.

11. Larson AC, Kellman P, Arai A, Hirsch GA, McVeigh E, Li D, Simonetti OP. Preliminary investigation of respiratory self-gating for free-breathing segmented cine MRI. Magn. Reson. Med. 2005; 53: 159-168.

12. Chapman B, Turner R, Ordidge RJ, Doyle M, Cawley M, Coxon R, Glover $\mathrm{P}, \mathrm{Mansfield} \mathrm{P}$. Real-time movie imaging from a single cardiac cycle by NMR. Magn. Reson. Med. 1987; 5: 246-254.

13. Eichenberger AC, Schwitter J, McKinnon GC, Debatin JF, von Schulthess GK. Phase-contrast echo-planar MR imaging: real-time quantification of flow and velocity patterns in the thoracic vessels induced by Valsalva's maneuver. J. Magn. Reson. Imaging 1995; 5: 648-655.

14. Riederer SJ, Wright RC, Ehman RL, Rossman J, Holsinger-Bampton E, Hangiandreou NJ, Grimm RC. Real-time interactive color flow MR imaging. Radiology 1991; 181: 33-39.

15. Klein C, Schalla S, Schnackenburg B, Bornstedt A, Fleck E, Nagel E. Magnetic resonance flow measurements in real time: Comparison with a standard gradient-echo technique. J. Magn. Reson. Imaging 2001; 14: 306-310.

16. Lee VS, Resnick D, Bundy JM, Simonetti OP, Lee P, Weinreb JC. Cardiac function: MR evaluation in one breath hold with realtime true fast imaging with steady-state precession. Radiology 2002; 222: 835-842.

17. Shankaranarayanan A, Simonetti OP, Laub G, Lewin JS, Duerk JL. Segmented k-space and real-time cardiac cine MR imaging with radial trajectories. Radiology 2001; 221: 827-836.

18. Nayak KS, Pauly JM, Kerr AB, Hu BS, Nishimura DG. Real-time color flow MRI. Magn. Reson. Med. 2000; 43: 251-258.
19. Nayak KS, Cunningham $\mathrm{CH}$, Santos JM, Pauly JM. Real-time cardiac MRI at 3 Tesla. Magn. Reson. Med. 2004; 51: 655-660.

20. Weiger M, Pruessmann KP, Boesiger P. Cardiac real-time imaging using SENSE. Magn. Reson. Med. 2000; 43: 177-184.

21. Pruessmann KP, Weiger M, Boesiger $P$. Sensitivity encoded cardiac MRI. J. Cardiovasc. Magn. Reson. 2001; 3: 1-9.

22. Körperich $\mathrm{H}$, Gieseke J, Barth $\mathrm{P}$, Hoogeween $\mathrm{R}$, Esdorn $\mathrm{H}$, Peterschröter A, Mezer $\mathrm{H}$, Beerbaum P. Flow volume and shunt quantification in pediatric congenital heart disease by real-time magnetic resonance velocity mapping. Circulation 2004; 109: 1987-1993.

23. Steeden JA, Atkinson D, Taylor AM, Muthurangu V. Assessing vascular response to exercise using a combination of real-time spiral phase contrast MR and noninvasive blood pressure measurements. J. Magn. Reson. Imaging 2010; 31: 997-1003.

24. Wintersperger BJ, Nikolaou K, Dietrich O, Rieber J, Nittka M, Reiser MF, Schoenberg SO. Single breath-hold real-time cine MR imaging: improved temporal resolution using generalized autocalibrating partially parallel acquisition (GRAPPA) algorithm. Eur. Radiol. 2003; 13: 1931-1936.

25. Tsao J, Kozerke S, Peter Boesiger P, Pruessmann KP. Optimizing spatiotemporal sampling for k-t BLAST and k-t SENSE: application to high-resolution real-time cardiac steady-state free precession. Magn. Reson. Med. 2005; 53: 1372-1382.

26. Uecker M, Hohage T, Block KT, Frahm J. Image reconstruction by regularized nonlinear inversion - joint estimation of coil sensitivities and image content. Magn. Reson. Med. 2008; 60: 674-682.

27. Uecker $M$, Zhang S, Frahm J. Nonlinear inverse reconstruction for real-time MRI of the human heart using undersampled radial FLASH. Magn. Reson. Med. 2010; 63: 1456-1462.

28. Uecker M, Zhang S, Voit D, Karaus A, Merboldt KD, Frahm J. Real-time magnetic resonance imaging at a resolution of $20 \mathrm{~ms}$. NMR Biomed. 2010; 23: 986-994.

29. Zhang S, Uecker M, Voit D, Merboldt KD, Frahm J. Real-time cardiovascular magnetic resonance at high temporal resolution: radial FLASH with nonlinear inverse reconstruction. J. Cardiovasc. Magn. Reson. 2010; 12: 39.

30. Zhang S, Block KT, Frahm J. Magnetic resonance imaging in real time: advances using radial FLASH. J. Magn. Reson. Imaging 2010; 31: 101-109.

31. Bernstein MA, Shimakawa A, Pelc NJ. Minimizing TE in moment-nulled or flow-encoded two- and three-dimensional gradient-echo imaging. J. Magn. Reson. Imaging 1992; 2: 583-588.

32. Bernstein MA, Grgic M, Brosnan TJ, Pelc NJ. Reconstructions of phase contrast, phased array multicoil data. Magn. Reson. Med. 1994; 32: 330-334.

33. Pelc NJ, Sommer FG, Li KC, Brosnan TJ, Herfkens RJ, Enzmann DR. Quantitative magnetic resonance flow imaging. Magn. Reson. Q. 1994; 10: 125-147.

34. Hundley WG, Li HF, Hillis LD, Meshacka BJ, Lange RA, Willard JE, Landau C, Peshock RM. Quantitation of cardiac output with velocity-encoded, phase-difference magnetic resonance imaging. Am. J. Cardiol. 1995; 75: 1250-1255.

35. Jung B, Honal M, Ullmann P, Hennig J, Markl M. Highly k-t-spaceaccelerated phase-contrast MRI. Magn. Reson. Med. 2008; 60: 1169-1177. 